\title{
PIKO-1, an effective, handy device for the patient's personal PEFR and FEV 1 electronic long-term monitoring
}

\author{
R.W. Dal Negro'1, C. Micheletto2, S. Tognella2, C. Turati1, R. Bisato1, \\ M. Guerriero 3 , M. Sandri4, P. Turco²
}

ABSTRACT: PIKO-1, an effective, handy device for the patient's personal PEFR and FEV $V_{1}$ electronic long-term monitoring. R.W. Dal Negro, C. Micheletto, S. Tognella, C. Turati, R. Bisato, M. Guerriero, M. Sandri, P. Turco.

Background and Aim. Subjects with airway obstruction are strongly recommended to monitor their lung function, which is particularly variable in asthma. Unlike PEFR, other personal measurements (such as $\mathrm{FEV}_{1}$ ) are still difficult to perform. PIKO-1 is the first electronic device for both PEFR and FEV 1 personal check, but its precision has not yet been assessed. The aim of this study was to compare PEFR and FEV 1 values from PIKO-1 and from a conventional spirometer in subjects with airway obstruction.

Methods. In total, 352 subjects $(217$ men; $47.6 \pm 19.0$ years; $72.6 \pm 15.0 \mathrm{~kg} ; 168.1 \pm 11.9 \mathrm{~cm})$ performed sequen- tial measurements using a PIKO-1 device and a spirometer. Wilcoxon signed-rank test and sign test were used as statistical tests.

Results. Mean $\mathrm{FEV}_{1}$ values from the spirometer and PIKO-1, respectively, were $2.9 \mathrm{~L} \pm 1.1$ and $3.0 \mathrm{~L} \pm 1.1$, and mean PEFR values were 466.1 L/min \pm 164.5 SD and 426.3 $\mathrm{L} / \mathrm{min} \pm$ 151.6 SD. PIKO-1 proved to overestimate $\mathrm{FEV}_{1}$ values by $4 \%(p<0.0001)$ and to underestimate PEFR values by $8 \%(p<0.000)$ systematically.

Conclusions. The precision of both PIKO-1 measurements (such as FEV 1 and PEFR) have been assessed. PEFR and FEV 1 measures should be reset by two different constants. Nevertheless, PIKO-1 is a suitable and reliable device for the personal monitoring of obstructive patients in real life.

Monaldi Arch Chest Dis 2007; 67: 2, 84-89.

1 Lung Dept., Orlandi Gen. Hosp., Bussolengo, Verona,

2 National Union for Asthma Patients,

${ }^{3}$ ESI Dept, Statistical Section,

4 CIDE Dept. University of Verona, Italy.

Correspondence: Roberto W. Dal Negro, Lung Dept., Orlandi General Hospital, Via Ospedale 2, 37012 Bussolengo, Verona, Italy; e-mail: rdalnegro@ulss22.ven.it

\section{Introduction}

Subjects with airway obstruction are strongly recommended to monitor their lung function, which is particularly variable in asthma [1-5]. Peak expiratory flow rate (PEFR) monitoring has been strongly recommended by asthma guidelines [1], and clinical studies [2] have shown that the routine use of peak flow meters, along with a self-management plan and education programme, can lead to a better control of asthma. Unlike PEFR, other spirometrical measurements (in particular $\mathrm{FEV}_{1}$ ) are difficult to perform regularly by asthma patients outside the lung clinic, the specialist outpatient department, or the GP's office, still being infrequent the use of appropriate and handy devices for simple lung function measurements to be performed at home, during work, or while travelling.

PIKO-1 is a recent electronic device for both PEFR and FEV 1 personal check: some of the most relevant characteristics claimed are pocket size ( $35 \mathrm{~g}$ weight; volume $7 \mathrm{~cm} \times 5 \mathrm{~cm} \times 1.5 \mathrm{~cm}$ ), with the possibility of setting predicted values (according to the ATS 1994 predicted values), the quality control of respiratory manoeuvres, the storage of several tests, and the precision of measurements.

PIKO-1 has been suggested as an adequate tool for both screening and monitoring [6]; nevertheless, the precision of measurements should be the crucial point that is faced when using simple technologies for critical measures that can highly affect the patient's management $[1,7-8]$. Since at the time of the study start-up no data as been available to our knowledge on this particular topic, the aim of the present study was to compare PEFR and $\mathrm{FEV}_{1}$ values obtained from the PIKO-1 device and from a conventional office spirometer in subjects with airway obstruction.

\section{Methods}

Main technical characteristics and potential of PIKO-1

As mentioned above, PIKO-1 (Pulmonary Data Services, Inc. - Ferraris Medical Ltd, Hertford, UK) is able to measure the patient's peak expiratory flow rate (PEFR) together with the patient's 
forced expiratory volume in one second $\left(\mathrm{FEV}_{1}\right)$. Additionally, PIKO-1 has a built-in memory which stores the values of the last 96 measurements performed, as well as both the corresponding date and time of their execution. It also stores and reports a comparison of each measurement to the patient's reference value for that parameter. The patient may review each of these measurements at any time, thus eliminating the need for clumsy hand-written and error-prone diaries. By using an integrated infrared port, this data can be downloaded from PIKO-1 to a personal computer, where a Windows-based tool allows physicians to monitor patients' lung function.

\section{Subjects}

Following their informed consent, 352 consecutive unselected subjects (217 males; mean age = 47.6 years $\pm 19.0 \mathrm{SD}$; mean weight $=72.6 \mathrm{~kg} \pm$ 15.0 SD; mean height $=168.1 \mathrm{~cm} \pm 11.9 \mathrm{SD}$ ) performed sequential measurements on a PIKO-1 device and on an office spirometer (Masterscreen, Jaeger, Germany) at the same time of the day (9-11 am). All patients used the same PIKO-1, and the mouthpiece was changed for each subject.

There were 259 asthma patients, with the remaining 93 subjects being affected with chronic obstructive pulmonary disease (COPD). Patients were characterised by different degrees of airway obstruction in baseline; 67 were current smokers; 112 were ex-smokers and the remaining 173 had never smoked. 244 patients regularly assumed respiratory drugs. Even though according to different treatment strategies of treatment, bronchodilators (mainly short- and long-acting $\beta_{2}$ adrenergics) used by the great majority of patients $(n=209)$, while anti-inflammatory drugs (mainly inhaled corticosteroids) were used by 136 subjects. The number of subjects assuming combined medication was 189, while 68 patients were without any active treatment. In order to avoid further causes of variability, all patients on treatment stopped their respiratory drugs 24 hours before both the PIKO-1 and the spirometrical tests. All patients performed both respiratory manoeuvres: $50 \%$ of patients first performed the PIKO-1 manoeuvre, while the other $50 \%$ first performed the spirometrical measure. The patients' compliance to both of the respiratory manoeuvres was assessed by an expert dedicated nurse.
The Masterscreen spirometer was calibrated daily before measurements in accordance with the internal calibration protocol, periodically checked and validated by the ISO 9001-2000 procedures for our lung department [9].

\section{Statistics}

The Wilcoxon signed-rank test and sign test were used.

The agreement between $\mathrm{FEV}_{1}$ and PEF measurements obtained by the PIKO- 1 and by the Masterscreen was assessed using the method proposed by Bland and Altman [10] and according to the recommendations of Westgard [11]. Analyses were undertaken using Stata 8.0 [12] and Matlab R13 [13].

\section{Results}

Spirometrical and PIKO-1 respiratory manoeuvres were correctly performed and easily obtained for all patients.

Mean $\mathrm{FEV}_{1}$ values from the Masterscreen spirometer and the PIKO-1, respectively, were 2.9 $\mathrm{L} \pm$ 1.1 SD and 3.0 L \pm 1.1 SD, while mean PEFR values were $466.1 \mathrm{~L} / \mathrm{min} \pm 164.5 \mathrm{SD}$ and 426.3 $\mathrm{L} / \mathrm{min} \pm$ 151.6 SD from the Masterscreen spirometer and the PIKO-1, respectively.

\section{Comparison between $F E V_{l^{-}}$sp and $F E V_{l} p k$}

The correlation between measurements was $0.983(95 \% \mathrm{CI}=0.979-0.986)$.

\section{Error-in-variable regression}

Varying the hypothesised reliability between 0.97 (3\% of noise variance on the total variance) and 1 (no measurement noise), the regression coefficient varied as shown in table 1: Bias calculated at the medical decision level $\mathrm{FEV}_{1}=3 \mathrm{~L}-0.109$ (95\% CI: -0.131 - -0.087).

Present data was shown to suggest that PIKO1 was overestimating $\mathrm{FEV}_{1}$ measurements systematically by $4 \%$. The standard deviation of the difference between measurements was 0.205.

More descriptive statistics for the differences are as follows: Skewness of the differences $=.26$; Kurtosis $=4.97$.

Figure 1 shows the following data:

a) Probability distribution of the differences;

b) The quartile plot of the differences;

c) Comparison plot: $\mathrm{FEV}_{1}$ measurements by PIKO vs spirometric measurements; and

Table 1. - FEV 1 measurements. Variability of regression coefficient by varying the hypothesised reliability between 0.97 ( $3 \%$ of noise variance on the total variance) and 1 (no measurement noise)

\begin{tabular}{lcccc}
\hline Reliability & Intercept & $\mathbf{9 5 \%}$ CI & Slope & 95\% CI \\
\hline 0.97 & -0.102 & $-0.124--0.081$ & 0.998 & $0.991-1.004$ \\
0.98 & -0.071 & $-0.112--0.031$ & 0.988 & $0.975-1.000$ \\
0.99 & -0.041 & $-0.094-0.011$ & 0.978 & $0.961-0.994$ \\
1.00 & -0.012 & $-0.073-0.050$ & 0.968 & $0.948-0.987$ \\
\hline
\end{tabular}


(a)

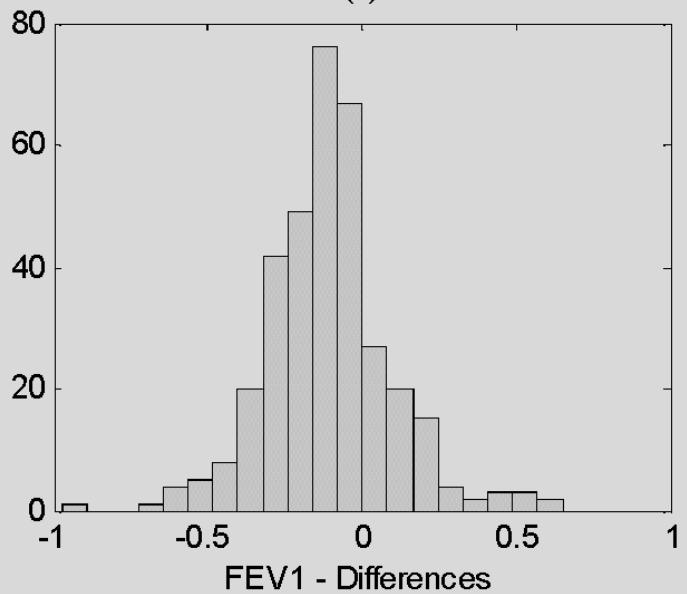

(c)

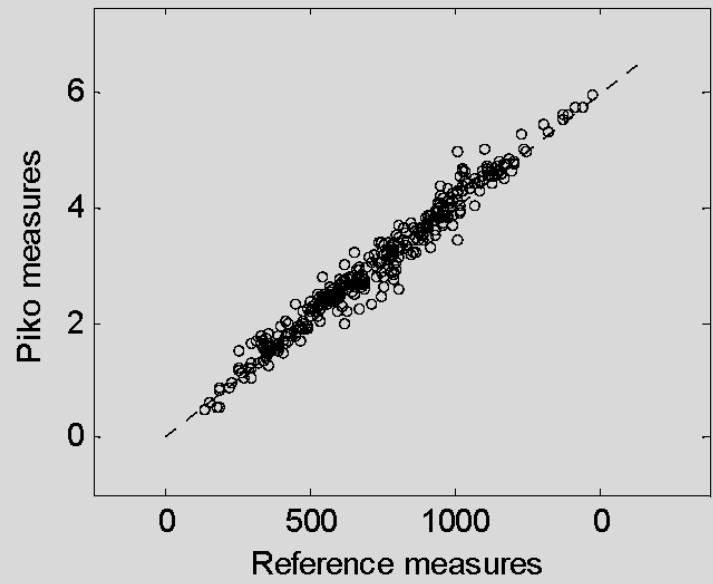

(b)

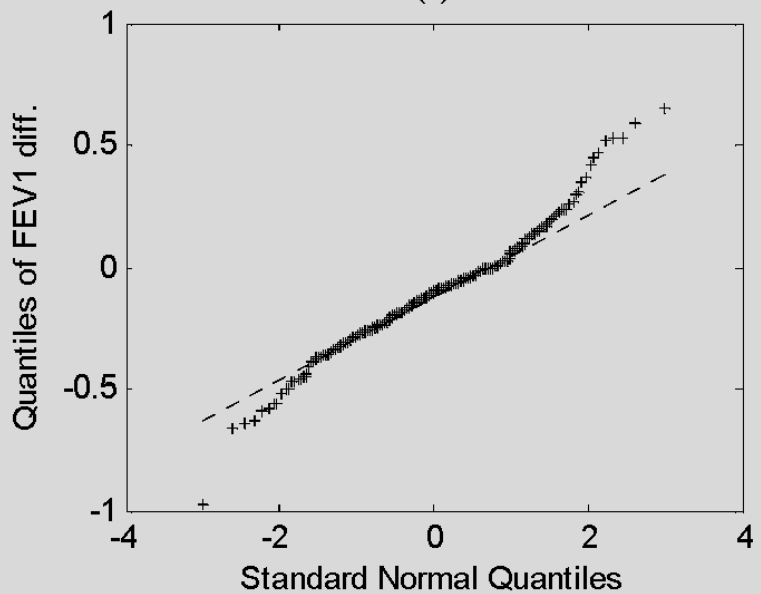

(d)

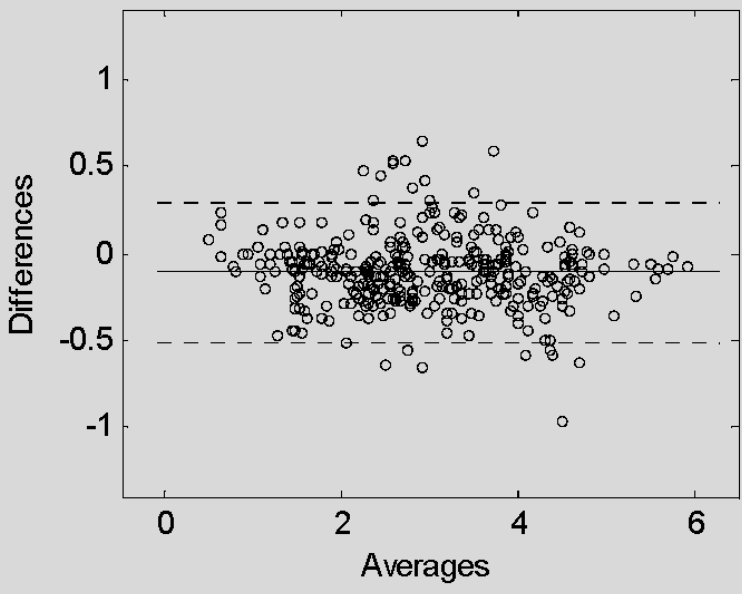

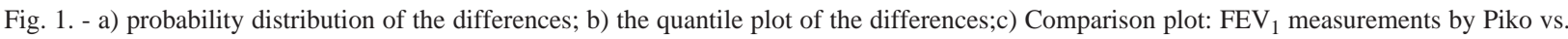
spirometric measurements; d) Bland-Altman plot of the differences together with the $95 \%$ limits of agreements.

d) Bland-Altman plot of the differences together with the $95 \%$ limits of agreement.

\section{Test for normality of the differences}

The following tests were performed:

a) Skewness/Kurtosis tests for normality resulted $\chi^{2}=19.46, p$-value $<0.001$

b) Shapiro-Wilk $W$ test for normal data resulted $\mathrm{z}$ $=4.64, p$-value $<0.001$.

These tests reject the normality hypothesis. Fig. 1 ( $a$ and $b$ ) indicates that the distance of the differences from the normal distribution is pronounced on the tails of distribution.

\section{Relationship between difference and magnitude}

See fig. 1 (d) and the following regressions. We first assessed the difference between the two methods based on the average of the two methods. Moreover, we assessed the absolute difference between the two methods based on the average of the two methods (tab. 2).

In terms of PEFR measurements, no relationship was proved between the difference of the measurements obtained from the two instruments and the extent of the same measurements. The trend of the error variability (such as the standard deviation) remained constant, even though the magnitude changed.

Table 2. - Parameters of the regression models for FEV 1 measures estimated by Deming regression for different levels of variance ratio. The error variability appears unaffected by the changes in magnitude of measures

\begin{tabular}{lcccc}
\hline & Intercept & $\mathbf{9 5 \%}$ CI & Slope & 95\% CI \\
\hline Difference & -0.062 & $-0.124--0.000$ & -0.016 & $-0.035-0.004$ \\
Absolute difference & 0.153 & $0.109-0.197$ & 0.009 & $-0.005-0.023$ \\
\hline
\end{tabular}




\section{Comparison between PEF-sp and PEF-pk}

The correlation between measurements was $0.943(95 \% \mathrm{CI}=0.930-0.953)$.

\section{Error-in-variable regression}

When varying the hypothesised reliability between 0.97 (3\% of noise variance on the total variance) and 1 (no measurement noise), the regression coefficient varied in the following manner (tab. 3):

Table 3. - Error-in-variable regression of PEFR measurements. Variability of regression coefficient by varying the hypothesised reliability between 0.97 (3\% of noise variance on the total variance) and 1 (no measurement noise)

\begin{tabular}{lcccc}
\hline Reliability & Intercept & 95\% CI & Slope & 95\% CI \\
\hline 0.90 & -21.31 & $-27.63--14.99$ & 1.14 & $1.13-1.56$ \\
0.93 & -5.59 & $-17.25-6.08$ & 1.10 & $1.08-1.13$ \\
0.96 & 9.15 & $-5.53-23.84$ & 1.07 & $1.04-1.10$ \\
1.00 & 27.43 & $10.08-44.78$ & 1.03 & $0.99-1.07$ \\
\hline
\end{tabular}
$=450$ was 54.84 . Kurtosis $=5.06$
Bias calculated at the medical decision level PEF

39.03 (95\% CI: 33.26 - 44.80) We can conclude that PIKO-1 was systematically underestimating PEFR measurements by $8 \%$. The standard deviation of the difference between measurements

More descriptive statistics for differences are as follows: Skewness of the differences $=0.98$;

Figure 2 shows the following data:

a) Probability distribution of the differences;

b) The quantile plot of the differences;

c) Comparison plot: PEFR measurements by PIKO vs. spirometric measurements; and

d) Bland-Altman plot of the differences together with the $95 \%$ limits of agreement.

(b)

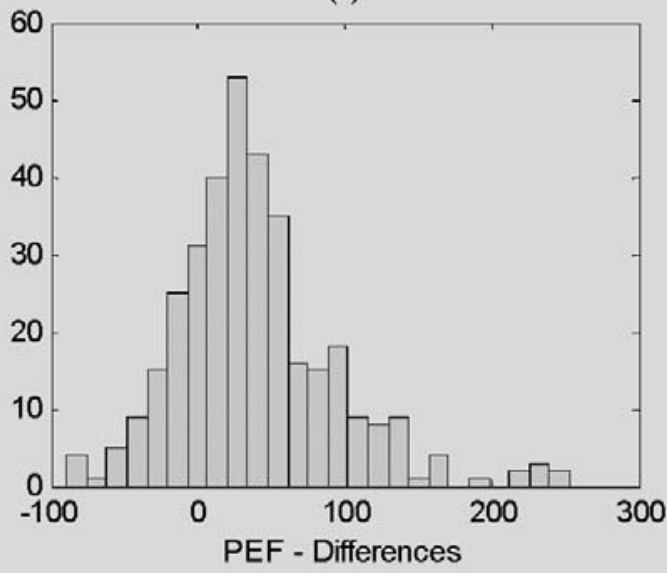

(c)

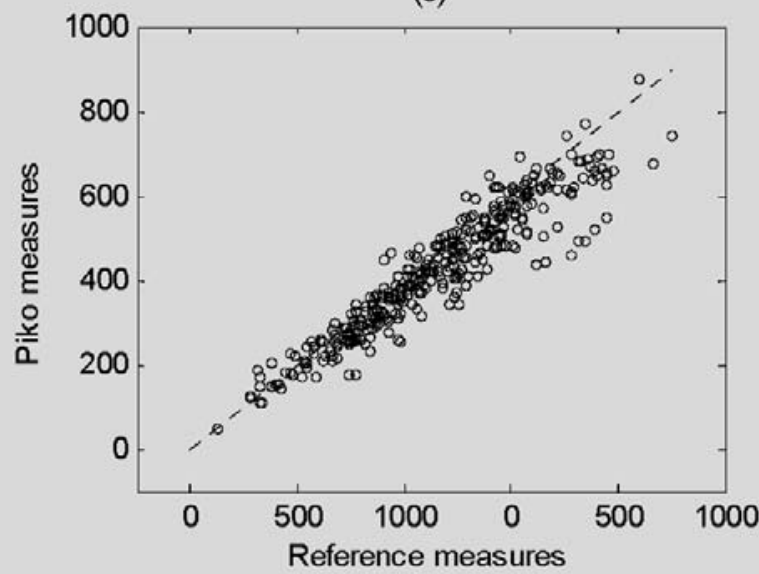

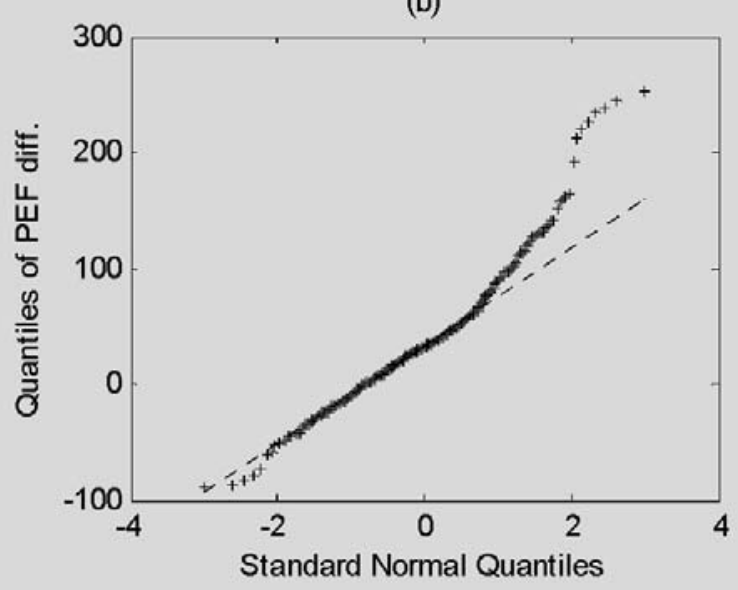

(d)

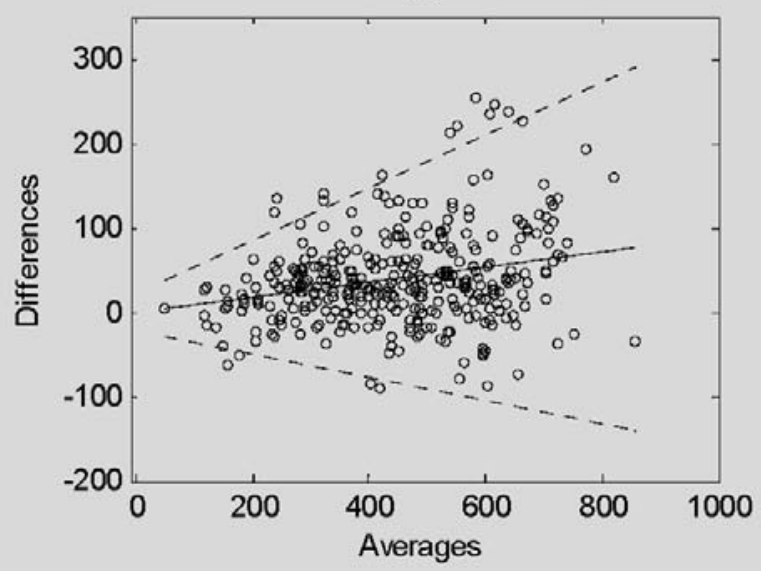

Fig. 2. - a) probability distribution of the differences; b)The quartile plot of the differences; c) Comparison plot: PEFR measurements by Piko vs. spirometric measurements; d) Bland-Altman plot of the differences together with the $95 \%$ limits of agreements. 


\section{Test for normality of the differences}

Skewness/Kurtosis tests for normality resulted $\chi^{2}=48.45, p$-value < 0.001; Shapiro-Wilk $W$ test for normal data resulted $\mathrm{z}=6.11, p$-value $<0.001$. These tests reject the normality hypothesis.

Figure $2 \mathrm{a}$ and $2 \mathrm{~b}$ indicate that the distance of the differences from the normal distribution is pronounced, in particular on the right tail of distribution.

\section{Relationship between difference and magnitude}

See fig. $2 \mathrm{~d}$ and the following regressions. We first assessed the difference between the two methods based on the average of the two methods.

Moreover, we assessed the absolute difference between the two methods on the average of the two methods (tab. 4; fig. 2d).

In terms of PEFR measurements, a relationship was proved between the differences in the measurements obtained from the two instruments and the extent of the measurements themselves. The bias and the variability of error (such as the standard deviation) increase by increased the magnitude.

\section{Discussion}

Regular (ideally daily) and long-term PEFR measurements are recommended for assessing changes in lung function and asthma severity, for monitoring response to therapeutic treatment, for evaluating the role of potential triggers, and for preventing respiratory exacerbations [1-2, 4].

Independently of the need for standardisation of the many different scales of PEFR charts now available [14], PEFR measurements are also partially biased by their intrinsic sensitivity and variability, which are not negligible in real life. If the former mainly depends on the large "effort dependence" of PEFR measurements in adults, the latter largely depends on the very low patient compliance and cooperation in the forced respiratory manoeuvres needed to perform PEFR measurements [7-8].

Spirometry also measures $\mathrm{FEV}_{1}$ (as the absolute value in litres) and $\mathrm{FEV}_{1} \%$ predicted, which is the most accepted indicator of the severity of airway obstruction [15-16]: $\mathrm{FEV}_{1}$ usually represents the most reliable and reproducible measurement for monitoring respiratory patients even though it is not as widely available as PEFR for the patient's personal check at home or outside the medical office [17-19]. At present, despite the recent great technological improvements in spirom- eters' engineering of recent years, $\mathrm{FEV}_{1}$ measurements are still regarded as possible to perform only in the presence of a conventional office spirometer, with all the related logistic and procedural limitations that need to overcome in real life [20].

PIKO-1 is a highly innovative, electronic instrument for easily detecting airway obstruction and for checking and monitoring both PEFR and $\mathrm{FEV}_{1}$ outside the medical office or clinic. It represents a substantial improvement in the self-management of obstructive patients, in particular of asthmatic patients, who can change their airway patency suddenly and can frequently need a rapid self-adjustment of drug assumption for controlling their respiratory disease.

This handy diagnostic device is based on the use of a patented flow-sensing mechanism. The core of this mechanism is a flat spring made out of a special alloy, developed for space applications, which offers stainless steel qualities and has very stable parameters up to $270{ }^{\circ} \mathrm{C}$. The pressure created by the air flow produces a deformation of the flat spring. The deformation is detected by some extensometers, whose resistance variation is measured through bridge technology. Since the deformation of the flat spring is proportional to the applied pressure which is related to the air flow according to a known function, it is possible to calculate the air flow itself. This calculation is performed by frequent measurements of the pressure exerted by the air flow. The measurement is in fact repeated every $1 / 100$ second.

The electronic section implementing the bridge technology mechanism also includes features for temperature compensation. In fact, the instrument has a self-compensation feature in the $0-50{ }^{\circ} \mathrm{C}$ interval. The accuracy of measurements is not affected by possible deposition of dirt on the surface of the spring; the mechanical parameters of the spring are the only factors in determining the computed pressure value. The only possible consequence of dirt deposition on the spring surface is variation of the offset at zero flow condition. However, this is taken into account by selfcalibration of the instrument when switched on, and hence the offset is subtracted from the following measurement operations. Only deposition of dirt disturbing the free movement of the spring could affect the reading and should be removed. However, this is unlikely, since it can occur only in the case of dirt deposition on the inner face of the spring, which is protected by the instrument case.

PIKO-1 has also proved to be resistant to ageing and environmental stress. As part of the FDA

Table 4. - In respect of the PEFR measurements, a relationship exists between the differences in measures obtained with the two instruments and the magnitude of measures

\begin{tabular}{lcccc}
\hline & Intercept & $\mathbf{9 5 \%}$ CI & Slope & 95\% CI \\
\hline Difference & -0.30 & $-17.38-16.78$ & 0.09 & $0.05-0.12$ \\
Absolute difference & 8.97 & $-4.88-22.82$ & 0.09 & $0.06-0.12$ \\
\hline
\end{tabular}


submission by the manufacturer, ten instrument units underwent 50,000 tests each, with a simulated air flow of $18 \mathrm{~L}$ per second. After this challenge, each unit was found to have deviated by only $0.1 \%$ from the initial readings.

The other relevant characteristics of PIKO-1, such as the fact that it is pocket size (35 g weight; volume $7 \times 5 \times 1.5 \mathrm{~cm}$ ); has the possibility of setting predicted values, and the quality control of respiratory manoeuvres, also are of great practical value for the effective self-management of patients with airway obstruction. PIKO-1 measurements proved easy to perform by all patients, independently of their original respiratory disease. This piece of evidence further supports the applicability of regular (or daily) self-check of airway patency' by all subjects, independently of their clinical setting. Even though systematically assessed, the distance between PIKO- 1 and spirometrical measurements were not so relevant in such an extent (such as $+4 \%$ and $-8 \%$ respectively) so as to limit their use in daily life, being the practical advantages highly consistent in terms of patients' management, without any possibility to mis-understand the patients' intrinsic severity.

In conclusion, although the office spirometer is an irreplaceable instrument for both detecting and managing patients with airway obstruction even in primary care, also the use of PIKO-1 should be regarded as a very good opportunity, being the implementation of $\mathrm{FEV}_{1}$ as a regular indicator of personal lung function to assess, register, and monitor easily, regularly (or daily) and inexpensively at home or when working the crucial improvement. Furthermore, when regularly adopted, PIKO-1 can also facilitate the patients' awareness of the disease and encourage their cooperation with the GP, or the pneumologist, in order to maintain their own clinical conditions under control.

\section{References}

1. GINA (Global Initiatives for Asthma) Workshop Report. Global strategy for asthma management and prevention. NIH Publication No. 02-3659, 2002.

2. Gibson PG, Powell H. Written action plans for asthma: an evidence-based review of the key components. Tho$\operatorname{rax} 2004$; 59: 94-9.

3. Mitchell DM, Gildeh P, Dimond AH. Value of serial peak expiratory flow measurements in assessing treat- ment response in chronic airflow limitation. Thorax 1986; 41: 606-10.

4. Asthma UK - Your personal asthma diary and action plan. National asthma Campaign. Hptt://www.asthma.org.uk/about/pdf/control.pdf (accessed july 2004).

5. Reddel H, Ware S, Marks G. Differences between asthma exacerbations and poor asthma control. Lancet 1999; 353: 364-9.

6. Fonseca JA, Costa-Pereira A, Delgado L, et al. Pulmonary function electronic monitoring devices. A randomized agreement study. Chest 2005; 128: 1258-1265.

7. Brusasco V. Usefulness of peak expiratory flow measurements: is it just a matter of instrument accuracy? Thorax 2003; 58: 375-6.

8. Verschelden P, Cartier A, L'Archeveque J. Compliance with and accuracy of daily self-assessment of peak expiratory flows (PEF) in asthmatic subjects over a three month period. Eur Respir J 1996; 9: 880-5.

9. Dal Negro RW, Farina M. Esempi applicativi del modello ISO 9001:2000. In "La Gestione per la Qualità in Pneumologia", Dal Negro R.W. \& Farina M. Eds., Springer Verlag Italia Publ., Milano, 2001, pagg. 55-128.

10. Bland JM, Altman DG. Measuring agreement in method comparison studies. Stat Methods Med Res 1999; 8: 135-60.

11. Westgard JO. Points of care in using statistics in method comparison studies. Clin Chem 1998; 44: 2240-2.

12. StataCorp (2001) Stata Statistical Software: Release 7.0. College Station, TX: Stata Corporation.

13. The Mathworks, Inc. (2002). Matlab. The language of technical computing. Natick, Massachusetts.

14. Reddel HK, Vioncent SD, Civitico J. The need of standardisation of peak flow charts. Thorax 2005; 60: 164-7.

15. American Thoracic Society. Lung function testing: selection of references values and interpretative strategies. Am Rev Respir Dis 1991; 144: 1202-18.

16. Expert Panel Report 2. Guidelines for the diagnosis and management of asthma. Clinical practice guidelines. National Institute of Health. Bethesda, Maylend, USA. Pub. No. 97-4051, 1997; 12-18.

17. Bellamy D, Hoskins G, Smith B. The use of spirometers in general practice. Asthma in general practice 1997; 5: 8-9.

18. Ferguson GT, Enright PL, Buist AS, Higgins MW. Office spirometry for lung health assessment in adults: a consensus statenet from the national lung Education Program. Respir Care 2002; 45: 513-30.

19. Wanger J, Irvin CG. Office spirometry: equipment selection and training of staff in the private practice setting. J Asthma 1997; 34: 93-104.

20. Enright PL, Studnicka M, Zielinski J. Spirometry to detect and manage chronic obstructive pulmonary disease and asthma in the primary care setting. Eur Respir Mon 2005; 31: 1-14.

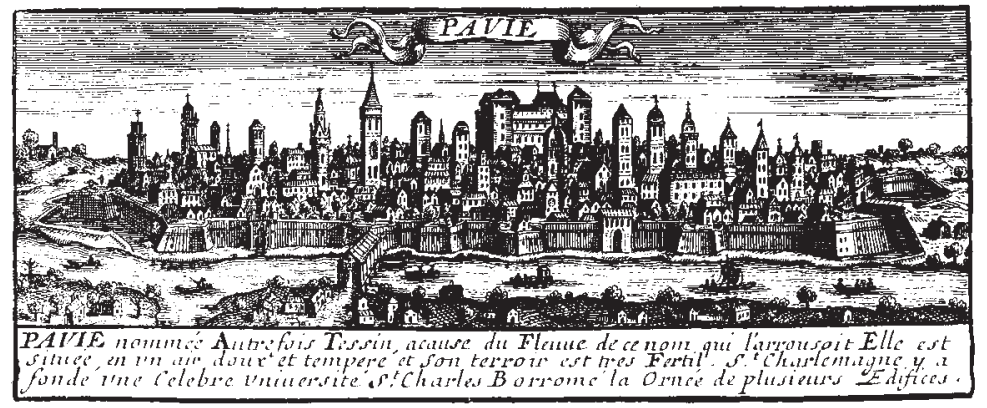

